\title{
A Parametric Analysis of the Basic Nonlinear Models of the Catalytic Reactions
}

\author{
V.I. Bykov*, S.B. Tsybenova \\ Emanuel Institute of Biochemical Physics, Russian Academy of Sciences, \\ ul. Kosygina 4, Moscow, 119334 Russia
}

\begin{abstract}
A series of the simplest thermokinetic models, in which the kinetic subsystem corresponds to traditional catalytic mechanisms, such as the Eley-Rideal mono- and bimolecular mechanisms and the Langmuir-Hinshelwood mechanism, and autocatalytic mechanisms characterized by multiplicity of steady states and autooscillations in the kinetic region, were considered. The method of continuation along a parameter was used for studying these basic models of thermokinetics. The parametric analysis performed allowed us to identify parameter regions characterized by critical effects (multiplicity of steady states and autooscillations).
\end{abstract}

Keywords and phrases: Chemical kinetics, catalysis, thermokinetics, bifurcation

Mathematics Subject Classification: 80A30, 34C60

\section{Introduction}

The interest to oscillating reactions of heterogeneous catalysis continues to this day [1-6]. These experimentally observed phenomena are interesting from both theoretical and practical points of view. They are characterized by a large set of physical and chemical factors which determine the conditions for of occurrence and development of chemical self-oscillations in such systems. In this work we consider some mathematical models of the nonlinear chemical kinetics in combination with a change in temperature of the reacting catalyst system.

As was shown in $[7,8]$, the kinetics of chain reactions can substantially influence nonisothermal modes of these processes. Competition between reaction chain branching and termination can play a predominant role in the formation of concentration chain propagation limits. This can be used to control detonation of mixtures by small chemically active admixtures.

On the other hand, a wide class of homogeneous-heterogeneous reactions are characterized by noticeable thermal effects [9-22]. For this reason, studies of the dynamics of chain and heterogeneous catalytic processes should generally be performed taking into account the special features of interactions between their kinetics and temperature dependences. Temperature and kinetic nonlinearities in combination can substantially complicate the physicochemical picture of processes. For instance, if the kinetic subsystem has several steady states, taking temperature changes into account leads to autooscillations, and, if

${ }^{*}$ Corresponding author. E-mail: vibykov@mail.ru 
the kinetic subsystem admits of autooscillations, the system as a whole can exhibit complex aperiodic regimes. Such nonlinear and nonstationary phenomena require the use of thermokinetic equations, which are systems of ordinary differential equations with parameters.

A study of solutions to these equations allows us to find the conditions of the appearance of critical phenomena, such as multiplicity of steady states, loss of stability by these states, the appearance of oscillation modes, etc. A special direction (a parametric analysis of the corresponding mathematical models) then appears in the context of the general mathematical modeling approach.

In this work, we consider a series of the simplest thermokinetic models, in which the kinetic subsystem corresponds to traditional catalytic mechanisms and autocatalytic mechanisms with multiplicity of steady states and autooscillations in the kinetic region [17-23]. Studies of the basic thermokinetic models were performed using the method of continuation along a parameter [24-28]. The parametric analysis performed allowed us to identify parameter regions with the existence of critical phenomena (multiplicity of steady states and autooscillations).

In the ideal mixing approximation, the thermokinetic model of a heterogeneous catalytic reaction can be written as

$$
\begin{aligned}
\frac{d T}{d t} & =\sum_{j=1}^{m} h_{j} w_{j}(T, \mathbf{x})+\alpha\left(T_{0}-T\right), \\
\frac{d x_{i}}{d t} & =\sum_{j=1}^{m} \gamma_{j i} w_{j}(T, \mathbf{x}), \quad i=1, \ldots, n,
\end{aligned}
$$

where $T$ is the temperature, $\mathbf{x}=\left(x_{1}, \ldots, x_{n}\right)$ is the vector of concentrations of intermediate substances adsorbed on the surface of the catalyst, $\gamma_{j i}$ denotes the stoichiometric coefficients of the reactions of the intermediates, $w_{j}$ is the rates of these reactions, $h_{j}$ is the heat effect of the $j$ th reaction per unit surface area, $\alpha$ is the coefficient characterizing the intensity of gas-solid heat exchange, and $t$ is the time. Model (1.1), (1.2) is simplified by ignoring reactions in the gas phase and the assumption that the concentrations of the substances observed are constant.

For a complex catalytic reaction, we have

$$
\sum_{i=1}^{n} \alpha_{i j} X_{i} \rightarrow \sum_{i=1}^{n} \beta_{i j} X_{i}, \quad j=1, \ldots, m,
$$

where $X_{i}$ denotes intermediate particles, $\alpha_{i j}$ and $\beta_{i j}$ are the stoichiometric coefficients, and $\gamma_{j i}=\beta_{i j}-\alpha_{i j}$. The rates of separate mechanism (1.3) stages, for instance, for the law of mass action, are written in the form

$$
\begin{aligned}
w_{j}(T, \mathbf{x}) & =k_{j}(T) \prod_{i=1}^{n} x_{i}^{\alpha_{i j}}, \\
k_{j}(T) & =k_{j}^{0} \exp \left(-\frac{E_{j}}{R T}\right),
\end{aligned}
$$

where $k_{j}, k_{j}^{0}$ and $E_{j}$ are the rate constant, preexponential factor, and activation energy of the $j$ th reaction, respectively, and $R$ is the universal gas constant.

In terms of dimensionless parameters and variables, thermokinetic model (1.1), (1.2) takes the form

$$
\begin{aligned}
& \frac{d T}{d t}=Q(T, \mathbf{x})+\alpha\left(T_{0}-T\right), \\
& \frac{d \mathbf{x}}{d t}=\mathbf{f}(T, \mathbf{x}),
\end{aligned}
$$

where $Q$ is the function characterizing heat release in the reaction on the surface of the catalyst and $\mathbf{f}$ is the vector function corresponding to the kinetics of transformations of intermediate substances. The 
separation of the heat balance equation (1.6) and kinetic subsystem (1.7)) corresponding to a complex chemical reaction on the surface of the catalyst is the special feature of dynamic system (1.6), (1.7).

It is well known $[9,11-19]$ that the kinetic subsystem itself (at $T \equiv$ const) can exhibit substantially nonlinear (multiplicity of steady states) and nonstationary (autooscillations) behaviors. For this reason, an important problem in an analysis of thermokinetic catalytic reaction models is studies of the special features of the interaction of the thermal and kinetic subsystems. Even the classic Semenov heat removalheat release diagram then has special features,

$$
\begin{aligned}
Q(T, \mathbf{x}) & =\alpha\left(T-T_{0}\right), \\
\mathbf{f}(T, \mathbf{x}) & =0,
\end{aligned}
$$

where the stationary heat balance equation is augmented by equations for the stationary state of intermediate substances. The elimination of unknown values $\mathbf{x}$ from (1.9) in the form $\mathbf{x}=\mathbf{x}(T)$ yields

$$
Q(T, \mathbf{x}(T))=\alpha\left(T-T_{0}\right),
$$

where the $\mathbf{x}(T)$ function in the case of multiple steady states can be characterized by a hysteresis. Moreover, the kinetic subsystem itself can be an oscillator. The interaction of the thermal and kinetic model constituents is then characterized by complex dynamic behavior. We studied the special features of such interactions for series of the so-called basic thermokinetic models, in which the heat balance equation is augmented by kinetic subsystems corresponding to standard mechanisms of catalytic transformations (the Eley-Rideal and Langmuir-Hinshelwood mechanisms, systems with autocatalysis, etc.). The use of the techniques of parametric analysis of dynamic systems allows fairly thorough analysis of the special features of interactions between the thermal and kinetic subsystems to be performed [24-28].

In this work, parametric analysis is performed in the simplest form for a series of so-called basic thermokinetic models of catalytic reactions. The models studied as a rule contain a minimum number of variables and variable parameters. Generally, the procedure of complete parametric analysis is a fairly laborious mathematical and computational problem. This work is limited to the initial parametric analysis stages. The results, however, give fairly abundant information about the possible dynamic behaviors of the thermokinetic models under consideration. In particular, they make it clear that the interaction of temperature nonlinearity with kinetic features of chemical reactions provides a diversity of dynamic characteristics of physicochemical processes. Moreover, parametric analysis allows the quantitative characteristics of chemical dynamics to be obtained, which is important not only theoretically but, especially, from the point of view of applications. For instance, in chemical technology of catalytic processes, it is important to know dangerous and safe boundaries of parameter variations, which can determine emergency or technologically undesirable regimes $[19,21]$.

\section{The Eley-Rideal monomolecular mechanism}

The scheme of transformations is as follows:

$$
\begin{aligned}
& \text { 1) } A+Z \rightarrow A Z, \\
& \text { 2) } B+A Z \rightarrow Z+A B,
\end{aligned}
$$

Here, $A, B$ and $A B$ are the gas-phase substances, $Z$ is the catalyst, and $A Z$ is the intermediate substance on its surface. The thermokinetic model (1.1), (1.2) that corresponds to scheme (2.1) has the form

$$
\begin{aligned}
& \frac{d T}{d t}=h_{1} w_{1}+h_{-1} w_{-1}+\alpha\left(T_{0}-T\right), \\
& \frac{d x}{d t}=w_{1}-w_{-1}-w_{2},
\end{aligned}
$$


where

$$
w_{1}=k_{1} p_{A} z, \quad w_{-1}=k_{-1} x, \quad w_{2}=k_{2} p_{B} x .
$$

For simplicity, we assume that only the second stage depends on temperature. The introduction of dimensionless parameters transforms (2.2) and (2.3) into the dimensionless model

$$
\begin{aligned}
& \frac{d x}{d \tau}=k_{1} p_{A} z-k_{-1} x-f(y) x, \\
& \frac{d y}{d \tau}=\beta f(y) x+s(1-y),
\end{aligned}
$$

where $z=1-x ; x$ and $y$ are the dimensionless concentration and temperature; $D a, \beta, \gamma, s, k_{1}, k_{-1}$ and $p_{A}$ are dimensionless parameters; and $f(y)=D a \exp (\gamma(1-1 / y))$ is the temperature dependence of the reaction rate.

Steady states of system (2.5), (2.6) are solutions to the equations

$$
\begin{aligned}
k_{1} p_{A} z-k_{-1} x-f(y) x & =0, \\
\beta f(y) x+s(1-y) & =0 .
\end{aligned}
$$

After the elimination of variable $y$, we obtain one equation for steady states,

$$
\beta f(y(x)) x+s(1-y(x))=0,
$$

where $y(x)=1+\frac{\beta}{s}\left(k_{1} p_{A}(1-x)-k_{-1} x\right)$.

Parametric dependences. For (2.7), dependences inverse to the sought dependences of steady states on parameters can explicitly be written as

$$
\begin{aligned}
D a(x) & =\frac{s(y(x)-1)}{\beta x \exp (\gamma(1-1 / y(x)))}, \\
\gamma(x) & =\frac{y(x)}{y(x)-1} \ln \frac{s(y(x)-1)}{\beta D a x} .
\end{aligned}
$$

To construct dependences of the stationary concentration and temperature on the other parameters, we must use numerical procedures of continuation with respect to parameters [22, 23]. Given these dependences, it is comparatively easy to construct the main bifurcation curves, $L_{\Delta}$ multiplicity and $L_{\sigma}$ neutrality curves. For instance, for the $D a$ and $\gamma$ parameters, after the subtraction of the elements of the Jacobi matrix for (2.5), (2.6),

$$
\begin{aligned}
& a_{11}=-k_{1} p_{A}-k_{-1}-f(y), \\
& a_{12}=-\frac{\gamma}{y^{2}} f(y) x, \\
& a_{21}=\beta f(y), \\
& a_{22}=\frac{\beta \gamma}{y^{2}} f(y) x-s .
\end{aligned}
$$

we obtain the following equations for the multiplicity and neutrality curves on the $(D a, \gamma)$ plane of parameters:

$$
\begin{aligned}
& L_{\Delta}(D a, \gamma):\left\{\begin{aligned}
\gamma(x) & =\frac{y^{2}(x)}{y(x)-1}\left(1+\frac{s(y(x)-1)}{\beta x\left(k_{1} p_{A}+k_{-1}\right)}\right), \\
D a & =D a(x, \gamma(x)) .
\end{aligned}\right. \\
& L_{\sigma}(D a, \gamma):\left\{\begin{aligned}
\gamma(x) & =\frac{y^{2}(x)}{y(x)-1}\left(1+\frac{(y(x)-1)}{\beta x}+\frac{\left(k_{1} p_{A}+k_{-1}\right)}{s}\right), \\
D a & =D a(x, \gamma(x)) .
\end{aligned}\right.
\end{aligned}
$$


(a)

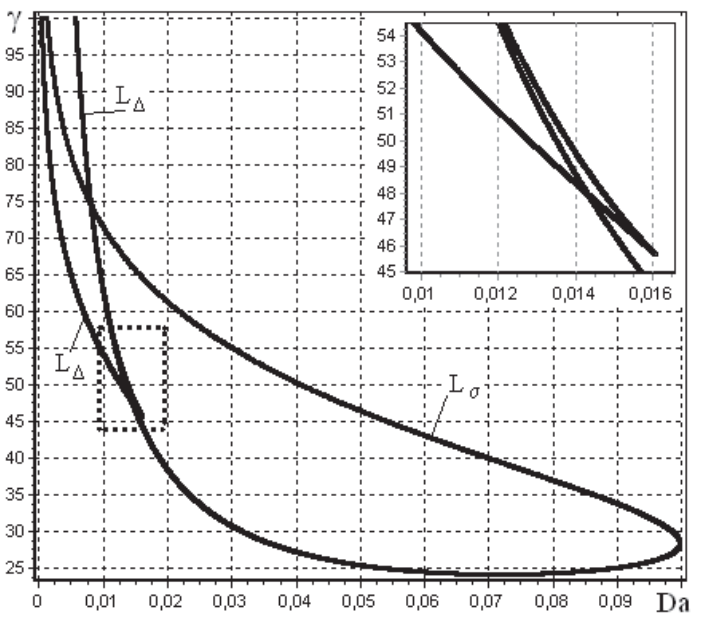

(b)

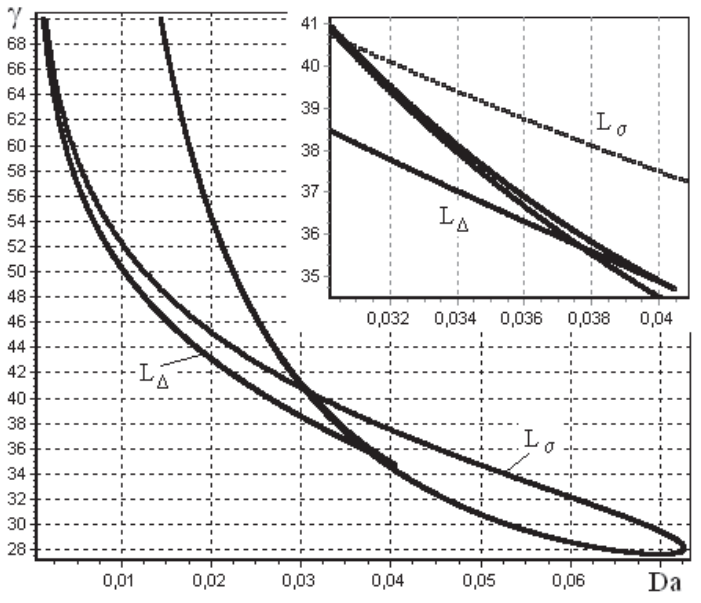

FiguRE 1. Stationary state multiplicity $\left(L_{\Delta}\right)$ and neutrality $\left(L_{\sigma}\right)$ curves for the EleyRideal (a) monomolecular and (b) bimolecular mechanisms: $\beta=$ (a) 0.8 and (b) 0.6 , $s=1, k 1=0.12, k_{-1}=0.01$, and $p_{A}=1$.

An example of the construction of bifurcation curves (2.10), (2.11) is given in Fig. 1a. The mutual arrangement of the bifurcation curves allows us to identify six parameter plane regions with different numbers and stabilities of steady states. For instance, over the parameter variation intervals $0.01 \leq$ $D a \leq 1$ and $25 \leq \gamma \leq 70$, there exists a fairly extended region with a single and unstable stationary state, which guarantees the presence of autooscillations in dynamic system (2.5), (2.6). Autooscillations can also exist at three steady states, but the corresponding region of parameters is fairly narrow. The developed procedure and program-mathematical software for parametric analysis of thermokinetic models of type (2.5), (2.6) can be used to study the influence of other factors on the conditions of the existence of critical effects. The bifurcation curves for the model specified were constructed at different parameter combinations, and the influence of a third parameter on the parametric portrait in the plane of two parameters was studied.

\section{The Eley-Rideal Bimolecular Mechanism}

Along with (11), one of the basic catalytic schemes of transformations is the following two-stage nonlinear mechanism of the oxidation of $C O$ :

$$
\begin{aligned}
& \text { 1) } \mathrm{O}_{2}+2 \mathrm{Z} \leftrightarrows 2 \mathrm{ZO}, \\
& \text { 2) } \mathrm{CO}+\mathrm{ZO} \rightarrow \mathrm{Z}+\mathrm{CO}_{2},
\end{aligned}
$$

The dimensionless thermokinetic model similar to (2.5), (2.6) that corresponds to this mechanism has the form

$$
\begin{aligned}
& \frac{d x}{d \tau}=2 k_{1} p_{A} z^{2}-2 k_{-1} x^{2}-f(y) x, \\
& \frac{d y}{d \tau}=\beta f(y) x+s(1-y),
\end{aligned}
$$

where the main denotations are the same as in (2.5), (2.6). The equation for steady states corresponding to $(3.2),(3.3)$ is similar to $(2.7)$, where

$$
y(x)=1+\frac{2 \beta}{s}\left(k_{1} p_{A}(1-x)^{2}-k_{-1} x^{2}\right) .
$$


The parametric dependences are similar to (2.8), (2.9) with the $y(x)$ function calculated according to (3.4). The Jacobi matrix elements for system (3.2), (3.3) are

$$
\begin{aligned}
& a_{11}=-4 k_{1} p_{A}(1-x)-4 k_{-1} x-f(y), \\
& a_{12}=-\frac{\gamma}{y^{2}} f(y) x \\
& a_{21}=\beta f(y) \\
& a_{22}=\frac{\beta \gamma}{y^{2}} f(y) x-s
\end{aligned}
$$

The stationary state multiplicity and neutrality curves in the $(D a, \gamma)$ plane of parameters have the form

$$
\begin{aligned}
& L_{\Delta}(D a, \gamma):\left\{\begin{aligned}
\gamma(x) & =\frac{y^{2}(x)}{y(x)-1}\left(1+\frac{s(y(x)-1)}{4 \beta x\left(k_{1} p_{A}(1-x)+k_{-1} x\right)}\right), \\
D a & =D a(x, \gamma(x)) .
\end{aligned}\right. \\
& L_{\sigma}(D a, \gamma):\left\{\begin{aligned}
\gamma(x) & =\frac{y^{2}(x)}{y(x)-1}\left(1+\frac{(y(x)-1)}{\beta x}+\frac{4}{s}\left(k_{1} p_{A}(1-x)+k_{-1} x\right)\right), \\
D a & =D a(x, \gamma(x)) .
\end{aligned}\right.
\end{aligned}
$$

An example of the construction of a parametric portrait for the Eley-Rideal bimolecular mechanism (3.1) is shown in Fig. 1b. As previously, we give only one parametric portrait variant, in the $(D a, \gamma)$ plane of parameters. The developed procedure and software can be used to fairly simply construct bifurcation curves in arbitrary parameter planes and study the influence of all the other parameters on their arrangement. This makes it possible to determine the critical conditions of the reaction and study the influence of various thermophysical and kinetic parameters on them. A comparative analysis of the parametric portraits for bimolecular and monomolecular mechanisms (Fig. 1a) shows that the regions of the existence of various regimes (multiplicity of steady states and autooscillations) depend on the kinetic characteristics of the mechanism of the reaction. Parametric analysis allows the quantitative characteristic of this influence to be obtained.

\section{The Langmuir-Hinshelwood Mechanism}

Let us consider the nonlinear three-stage scheme of transformations

$$
\begin{aligned}
& \text { 1) } A+2 Z \leftrightarrows 2 A Z, \\
& \text { 2) } B+Z \leftrightarrows B Z, \\
& \text { 3) } A Z+B Z \rightarrow 2 Z+A B
\end{aligned}
$$

where $Z$ is the catalyst and $A Z$ and $B Z$ are intermediate substances. The corresponding dimensionless thermokinetic model has the form

$$
\begin{aligned}
\frac{d x_{1}}{d \tau} & =2 k_{1} p_{A} z^{2}-2 k_{-1} x_{1}^{2}-f(y) x_{1} x_{2}, \\
\frac{d x_{2}}{d \tau} & =k_{2} p_{B} z-k_{-2} x_{2}-f(y) x_{1} x_{2} \\
\frac{d y}{d \tau} & =\beta f(y) x_{1} x_{2}+s(1-y)
\end{aligned}
$$

where the kinetic subsystem is nonlinear. It is well known [11] that, under isothermal conditions, system (4.2), (4.3) is a catalytic trigger. It contains three steady states, two of which are stable, and one, unstable. The dependence of the stationary rate of the third reaction can therefore have a hysteresis with 
respect to the $y$ parameter; that is, in the stationary state, the heat release function in stationary heat balance equation (4.4),

$$
\beta f(y) x_{1}(y) x_{2}(y)+s(1-y)=0
$$

can have a hysteresis character. Generally, it is difficult to obtain the corresponding explicit equations, but, in the particular case of irreversible adsorption stages in (5.1), a visual geometric interpretation can be given. Indeed, for $k_{-1}=k_{-2}=0$, we find from (4.2), (4.3) that

$$
\begin{aligned}
2 k_{1} p_{A} z^{2}-f(y) x_{1} x_{2} & =0 \\
k_{2} p_{B} z-f(y) x_{1} x_{2} & =0 .
\end{aligned}
$$

System (4.6), (4.7) has four solutions at $y=$ const, two boundary solutions, (1) $x_{1}=1, x_{2}=0$ and (2) $x_{1}=0, x_{2}=1$, for which the rate of the reaction is zero, and, at certain parameter values, two steady states, for which

$$
z=\frac{k_{2} p_{B}}{2 k_{1} p_{A}}
$$

The $x_{1}$ and $x_{2}$ values are determined by solving the quadratic equation and can be found in the explicit form. We do not use and therefore do not give it here. According to (4.8), Semenov's diagram (4.5) in the steady state has the form

$$
\frac{\left(k_{2} p_{B}\right)^{2}}{2 k_{1} p_{A}}=s(y-1) .
$$

In addition to (4.8), the zero branch of the stationary reaction rate should be taken into account; the stationary temperature for it is $y=1$. Calculations show that, at small $k_{-1}$ and $k_{-2}$, the temperature dependence of the stationary reaction rate changes insignificantly except the bifurcation points of jumps from one branch of stable steady states to the other. The stationary values can differ from (4.9) insignificantly.

Thermokinetic model (4.2)-(4.4) is a three-dimensional dynamic system. It combines a trigger and an oscillators, which is its special feature. The kinetic subsystem has trigger properties, and the "temperature +concentration" subsystem can have autooscillations. The dynamics of system (4.2)-(4.4) can generally be fairly complex, and its details require a special study.

\section{Autocatalytic Transformation Schemes}

We here study several thermokinetic models corresponding to autocatalytic reaction schemes. They are in a certain sense the simplest nonlinear schemes with essentially nonlinear properties (multiplicity of steady states and autooscillations). In isothermal formulation, the corresponding kinetic models are considered in $[9,11]$. The special feature of this study is a combination of temperature and kinetic nonlinearities in thermokinetic models, which can considerably complicate the observed physicochemical picture of catalytic reactions with substantial heat effects. Let us consider the autocatalytic system

$$
\begin{aligned}
& \text { 1) } Z \leftrightarrows X \\
& \text { 2) } X+2 Z \rightarrow 3 Z
\end{aligned}
$$

where $Z$ is the active site of the surface of the catalyst and $X$ is the intermediate compound. The thermokinetic model that corresponds to scheme (5.1) is as follows:

$$
\begin{aligned}
& \frac{d T}{d t}=\sum_{i} h_{i} w_{i}(x, T)+\alpha\left(T_{0}-T\right), \\
& \frac{d x}{d t}=w_{1}(x, T)-w_{-1}(x, T)-w_{2}(x, T),
\end{aligned}
$$


where

$$
\begin{aligned}
w_{1}(x, T) & =k_{1}(T)(1-x), \\
w_{-1}(x, T) & =k_{-1}(T) x, \\
w_{2}(x, T) & =k_{2}(T) x(1-x)^{2} .
\end{aligned}
$$

Let us introduce dimensionless variables and parameters using the Frank-Kamenetskii scheme for this purpose and assume for simplicity that only $k_{-1}$ depends on temperature $\left(E_{1}=E_{2}=0, h_{1}=h_{2}=0\right.$, $\left.E_{-1} \neq 0, h_{-1} \neq 0\right)$. System (5.2) can then be written as

$$
\begin{aligned}
& \frac{d y}{d \tau}=\beta f(y) x+s(1-y)=f_{1}(x, y), \\
& \frac{d x}{d \tau}=k_{1} z-f(y) x-k_{2} x z^{2}=f_{2}(x, y),
\end{aligned}
$$

where $z=1-x ; f(y)=D a \exp (\gamma(1-1 / y))$ (as previously); $x$ and $y$ are the dimensionless concentration and temperature, respectively; $\beta, s, D a, \gamma, k_{1}$, and $k_{2}$ are dimensionless parameters; and $\tau$ is the dimensionless time. As mentioned, our goal is to find the region of autooscillations, study the influence of various parameters on the characteristics of the system, determine the dynamics of the process, and divide the parametric space into regions corresponding to qualitatively different dynamic behavior types.

The steady states of system (5.3) are the solutions to the equations

$$
\begin{aligned}
\beta f(y) x+s(1-y) & =0, \\
k_{1} z-f(y) x-k_{2} x z^{2} & =0 .
\end{aligned}
$$

Eliminating $y$ and substituting it into the first equation, we obtain the nonlinear equation

$$
\beta D a \exp (\gamma(1-1 / y(x))) x+s(1-y(x))=0,
$$

where $y(x)=1+\left(k_{1}-k_{2} x(1-x)\right)(1-x) \beta / s$.

The type of steady state stability is determined by the roots of the characteristic equation

$$
\lambda^{2}-\sigma \lambda+\Delta=0,
$$

where $\sigma$ and $\Delta$ are found using the Jacobi matrix elements

$$
\begin{aligned}
& a_{11}=\frac{\partial f_{1}}{\partial y}=\frac{\gamma}{y^{2}} \beta x f(y)-s, \\
& a_{12}=\frac{\partial f_{1}}{\partial x}=\beta f(y), \\
& a_{21}=\frac{\partial f_{2}}{\partial y}=-\frac{\gamma}{y^{2}} x f(y), \\
& a_{22}=\frac{\partial f_{2}}{\partial x}=-k_{1}-f(y)-k_{2}(1-x)(1-3 x),
\end{aligned}
$$

which gives

$$
\begin{aligned}
\sigma & =a_{11}+a_{22}, \\
\Delta & =a_{11} a_{22}-a_{12} a_{21} .
\end{aligned}
$$

Parametric dependences. Steady-state equation (5.4) can be used to obtain parametric dependences in the explicit form, for instance,

$$
D a(x)=\frac{s(y(x)-1)}{\beta x \exp (\gamma(1-1 / y(x)))} .
$$




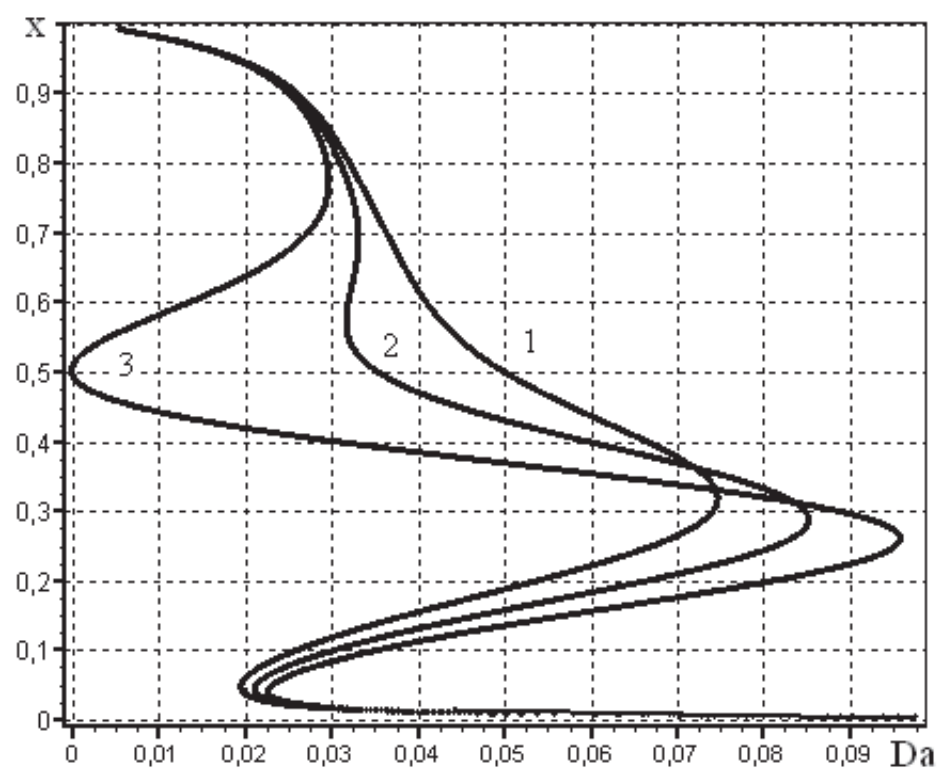

FiguRE 2. $x(D a)$ parametric dependences for an autocatalytic trigger: $\beta=0.375, s=2$, $\gamma=75, k_{1}=(1) 2$, (2) 2.2 , and (3) 2.4 .

Calculations show that the region of multiplicity of steady states decreases as the $s$ parameter increases. Similarly, we can obtain the $\gamma(x)$ dependence

$$
\gamma(x)=\frac{y(x)}{y(x)-1} \ln \frac{s(y(x)-1)}{\beta x D a} .
$$

In this plane of parameters, the region of multiplicity decreases as $\beta$ increases (Fig. 2). Figure 2 contains only one example of the construction of parametric dependences for steady states. The $x(D a)$ plot shows that, over the interval $0.02 \leq D a \leq 0.03$, five steady states can exist on the third curve. As the second parameter is varied, the intervals of the existence of three and five steady states change. The presence of five steady states in this model corresponds to the interaction of kinetic nonlinearity (under isothermal conditions, the autocatalytic process under consideration is a trigger) with temperature nonlinearity.

Multiplicity curves $L_{\Delta} . \Delta=0$ is the boundary separating the region of parameters into regions with one and three steady states. For instance, let us consider the $(D a, \gamma)$ plane of parameters and write an equation for the $L_{\Delta}$ multiplicity curve in this plane. For this purpose, we must solve the system

$$
\begin{aligned}
& f_{1}(x, y, D a, \gamma)=0 \\
& f_{2}(x, y, D a, \gamma)=0 \\
& \Delta(x, y, D a, \gamma)=0
\end{aligned}
$$

where $\Delta$ is determined using Jacobi matrix elements (5.6).

Using explicit equation (5.8) for $D a(y, \gamma)$ and substituting it into (5.7), we obtain the boundaries of the region of multiplicity of steady states in the $(D a, \gamma)$ plane of parameters,

$$
L_{\Delta}(D a, \gamma):\left\{\begin{aligned}
\gamma(x) & =\frac{y(x)^{2}}{y(x)-1}\left(1+\frac{s(y(x)-1)}{\beta x\left(k_{1}+k_{2}(1-x)(1-3 x)\right)}\right) \\
D a & =D a(x, \gamma(x)) .
\end{aligned}\right.
$$




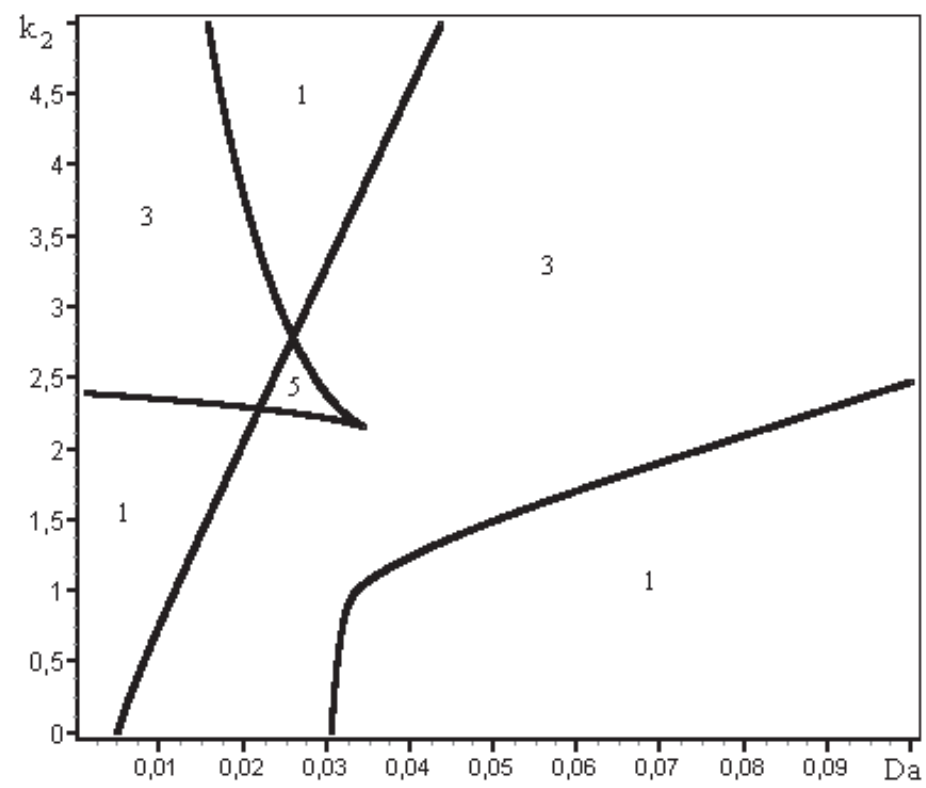

Figure 3. Multiplicity curves $L_{\Delta}\left(k_{2}, D a\right)$ for model (5.3) with five steady states; numbers $1-5$ correspond to the numbers of states in the corresponding parameter regions; $\beta=0.375, s=2, \gamma=75$, and $k_{1}=0.6$.

An example of multiplicity curves constructed using explicit equations in the $\left(D a, k_{2}\right)$ plane of parameters is given in Fig. 3. The region with five steady states is fairly narrow, whereas three steady states are observed over a wide region of parameters, and this region consists of two parts, which correspond to two types of nonlinearities present in the model under consideration, temperature and kinetic nonlinearities.

The dependences obtained can be used to fairly simply study changes in the multiplicity region in various parameter planes. The calculation results show that the multiplicity region increases in the $(D a, \gamma)$ parameter plane as the $s$ parameter decreases.

We were unable to obtain explicit equations for $L_{\Delta}$ in other parameter planes. For this reason, we used the procedure for graphically constructing $L_{\Delta}$.

This procedure involves the construction of a series of parametric dependences with respect to one of the parameters while the second parameter is varied. Note that we were able to construct the corresponding dependences for all the parameters. The region of multiplicity of steady states lies between the turning points of parametric curves (their maximum and minimum values). As the second parameter is varied, the boundaries of this region limit the region of multiplicity of steady states on the corresponding parameter plane.

Neutrality curves $L_{\sigma} . \sigma=0$ determines the type of stability of steady states. To construct $L_{\sigma}$, for instance, in the $(D a, \gamma)$ plane, we must solve the system of equations

$$
\begin{aligned}
f_{1}(x, y, D a, \gamma) & =0 \\
f_{2}(x, y, D a, \gamma) & =0 \\
\sigma(x, y, D a, \gamma) & =0 .
\end{aligned}
$$




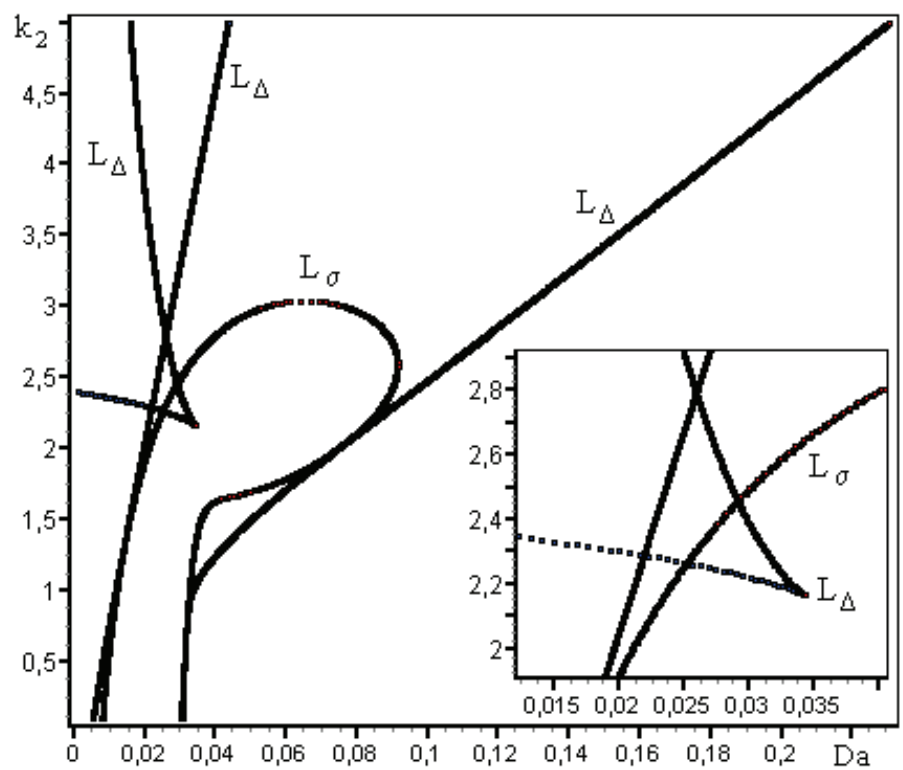

Figure 4. Parametric portrait of model (5.3) on the $\left(D a, k_{2}\right)$ plane: $\beta=0.375, s=2$, $\gamma=75$, and $k_{1}=0.6$.

Substituting the explicit equation for the $D a(x)$ parametric dependence into the third equation yields the neutrality curve equation in the explicit form

$$
L_{\sigma}(D a, \gamma):\left\{\begin{aligned}
\gamma(x) & =\frac{y(x)^{2}}{y(x)-1}\left(1+\frac{y(x)-1}{\beta x}+\frac{k_{1}+k_{2}(1-x)(1-3 x)}{s}\right), \\
D a & =D a(x, \gamma(x)) .
\end{aligned}\right.
$$

It was difficult to obtain explicit equations for constructing $L_{\sigma}$ in the other parameter planes. For this reason, we had to use a graphic procedure for constructing neutrality curves too. We constructed series of parametric dependences with respect to one of the parameters and varied the second parameter. Values corresponding to $\sigma$ sign change, that is, $\sigma=0$, were plotted on the parameter plane.

The mutual arrangement of multiplicity $\left(L_{\Delta}\right)$ and neutrality $\left(L_{\sigma}\right)$ curves determines the parametric portrait of the system. It describes various parameter regions with different stationary state numbers and stability types. In the system under consideration, there can be six such regions (see Fig. 4), including regions (1) with a single stable stationary state, (2) with a single unstable stationary state, (3)-(5) three steady states two of which can be stable, (6) three unstable steady states, an so on. As previously, the region bounded by the multiplicity curve consists of two subregions, and the loop of the neutrality curve is situated between them.

\section{An Autocatalytic Oscillator}

Let us augment scheme (5.1) by the so-called buffer stage,

1) $Z \leftrightarrows X_{1}$

2) $X_{1}+2 Z \rightarrow 3 Z$

3) $Z \leftrightarrows X_{2}$, 
where $X_{1}$ and $X_{2}$ are intermediate substances on the surface of catalyst $Z$. As is known, the kinetic model corresponding to autocatalytic system of transformations (6.1) is the simplest autocatalytic oscillator (a system that admits of autooscillation modes). As previously, let us augment kinetic model (6.1) by a heat balance equation and consider the resulting system in the dimensionless form

$$
\begin{aligned}
\frac{d y}{d \tau} & =\beta f(y) x_{1} z^{2}+s(1-y), \\
\frac{d x_{1}}{d \tau} & =k_{1} z-k_{-1} x_{1}-f(y) x_{1} z^{2}, \\
\frac{d x_{2}}{d \tau} & =k_{3} z-k_{-3} x_{2},
\end{aligned}
$$

where $z=1-x_{1}-x_{2} ; x_{1}$ and $x_{2}$ are the dimensionless concentrations and temperature; and $D a, \beta, \gamma$, $s, k_{1}, k_{-1}, k_{3}$, and $k_{-3}$ are the dimensionless parameters of the system.

For system (6.2), steady states are found using the equation

$$
\beta f\left(y\left(x_{1}\right)\right) x_{1} z^{2}+s\left(1-y\left(x_{1}\right)\right)=0,
$$

with respect to $x_{1}$. Here,

$$
\begin{aligned}
x_{2} & =\frac{k_{3}\left(1-x_{1}\right)}{k_{3}+k_{-3}} ; \\
\alpha & =\frac{k_{3}}{k_{3}+k_{-3}} ; \\
z & =\left(1-x_{1}\right)(1-\alpha) ; \\
y\left(x_{1}\right) & =1+\frac{\beta}{s}\left(k_{1} z-k_{1} x_{1}\right) ; \\
f\left(y\left(x_{1}\right)\right) & =D a \exp (\gamma(1-1 / y)) .
\end{aligned}
$$

Steady state stability conditions. There are many criteria that can be used to estimate the sign of Jacobi matrix eigenvalues without calculating the roots themselves. One of these is the Rauss-Hurwitz criterion. For instance, for system (6.2) of three equations, it can be formulated as follows. The thirdorder characteristic equation has the form

$$
\lambda^{3}-\sigma \lambda^{2}+\delta \lambda-\Delta=0
$$

where $\sigma, \Delta$, and $\delta$ are expressed in terms of the Jacobi matrix elements.

According to the Rauss-Hurwitz criterion, a stationary state is stable if the inequalities $\sigma>0, \theta=$ $\sigma \delta-\Delta>0$, and $\Delta>0$ are satisfied. The Jacobi matrix elements for system (6.2) are

$$
\begin{aligned}
& a_{11}=-\left(k_{1}+k_{-1}\right)-f(y)\left(z-2 x_{1}\right), \\
& a_{12}=-k_{1}+2 f(y) x_{1} z, \\
& a_{13}=-\frac{\gamma}{y^{2}} f(y) x_{1} z^{2}, \\
& a_{21}=-k_{3} \\
& a_{22}=-\left(k_{3}+k_{-3}\right) \\
& a_{23}=0 \\
& a_{31}=\beta f(y) z\left(z-2 x_{1}\right) \\
& a_{32}=-2 \beta f(y) x_{1} z, \\
& a_{33}=\frac{\gamma}{y^{2}} \beta f(y) x_{1} z^{2}-s .
\end{aligned}
$$


(a)
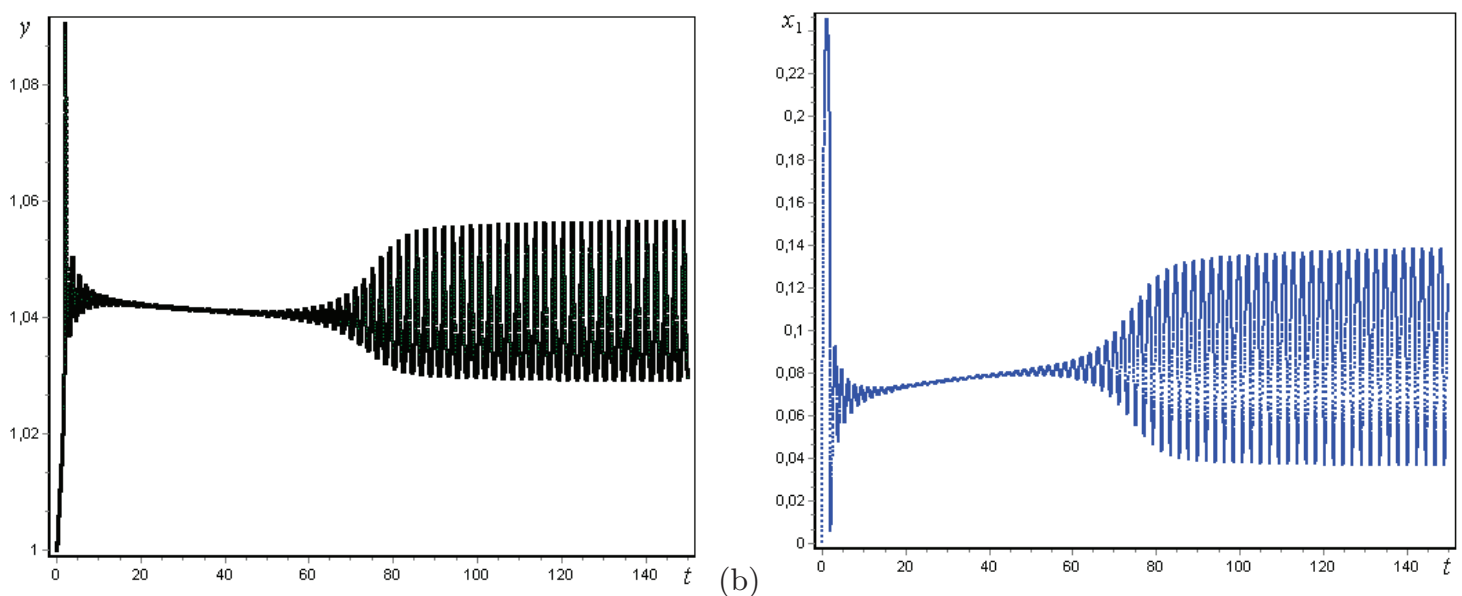

Figure 5. Time dependences of solutions to system (6.2) at $\gamma=65, \beta=0.4, s=4.5$, $D a=0.56, k_{1}=0.6, k_{-1}=1, k_{3}=0.001, k_{-3}=0.03, x_{1}(0)=x_{2}(0)=0, y(0)=1$; (a) $y(t)$ and $(\mathrm{b}) x_{1}(t)$.

As with the models considered above, the steady state conditions for system (6.2) can be used to obtain explicit equations for parametric dependences,

$$
\begin{aligned}
D a\left(x_{1}\right) & =\frac{s}{\beta} \frac{y\left(x_{1}\right)-1}{x_{1} z^{2} \exp \left(\gamma\left(1-1 / y\left(x_{1}\right)\right)\right)}, \\
\gamma\left(x_{1}\right) & =\frac{y\left(x_{1}\right)}{y\left(x_{1}\right)-1} \ln \frac{s\left(y\left(x_{1}\right)-1\right)}{\beta \operatorname{Dax} z_{1} z^{2}} .
\end{aligned}
$$

As previously, if we know the explicit parametric dependence form, we can perform a parametric analysis of model (6.2) and comparatively easily construct bifurcation curves that determine multiplicity of steady states, their stability, and the conditions of the existence of autooscillations. Examples of numerically constructing solutions for thermokinetic oscillator (6.2) and its parametric analysis are given in Figs. 5-7. Of interest are two types of thermokinetic oscillations shown in Figs. 5 and 7.

Self-oscillations of the first type arise when parameters responsible for oscillations are, as previously. Selected in the concentration-temperature phase plane $x, y$. In this situation, there can be the so-called induction period of arising of oscillation. The system nonmonotonically goes to the mode of stable oscillations. Such oscillations are observed when the $D a$ parameter ranges from 0.4975 to 0.568 .

The parametric portrait of model (6.2), as distinct from the models considered above, consists of three curves $\sigma=0, \Delta=0$, and $\theta=0$. For instance, in the $(D a, \beta)$ plane of parameters, these curves divide the parametric plane into a large number of regions with different stationary state numbers and stability types (Fig. 8).

To summarize, a combination of kinetic and temperature nonlinearities can lead to considerable complication of the dynamics of a catalytic system. For instance, if there is multiplicity of steady states in the kinetic subsystem, temperature changes can cause autooscillations or a complex dynamics if there are three degrees of freedom. The mathematical models considered are in a certain sense simplest, they are minimum in both nonlinearity and phase space dimension. In thermal-kinetic oscillators, the nonlinearity of the kinetic component does not exceed three, and the temperature dependence is traditionally exponential, $\exp (\gamma(1-1 / y(x)))$. Our data show that the suggested models can be used as a basis for the description and interpretation of a complex dynamics of thermokinetic processes on the surface of catalysts. 


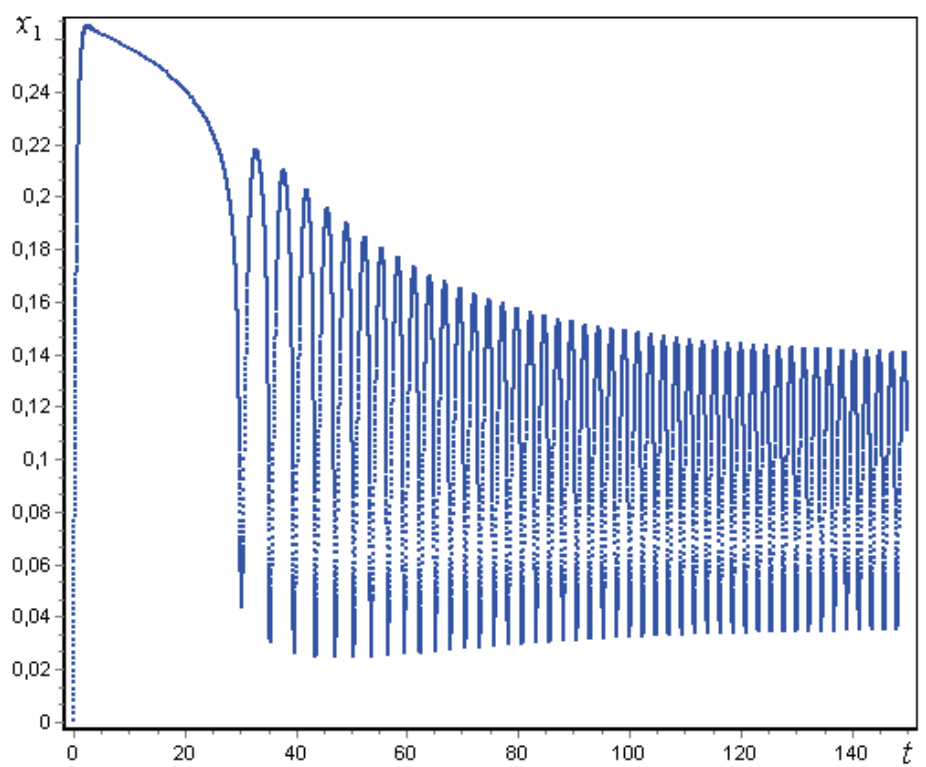

Figure 6. Time dependences of solutions to system (6.2) at $\gamma=65, \beta=0.4, s=4.5$, $D a=0.56, k_{1}=0.6, k_{-1}=1, k_{3}=0.001, k_{-3}=0.03, x_{1}(0)=0, x_{2}(0)=0.1, y(0)=1$.

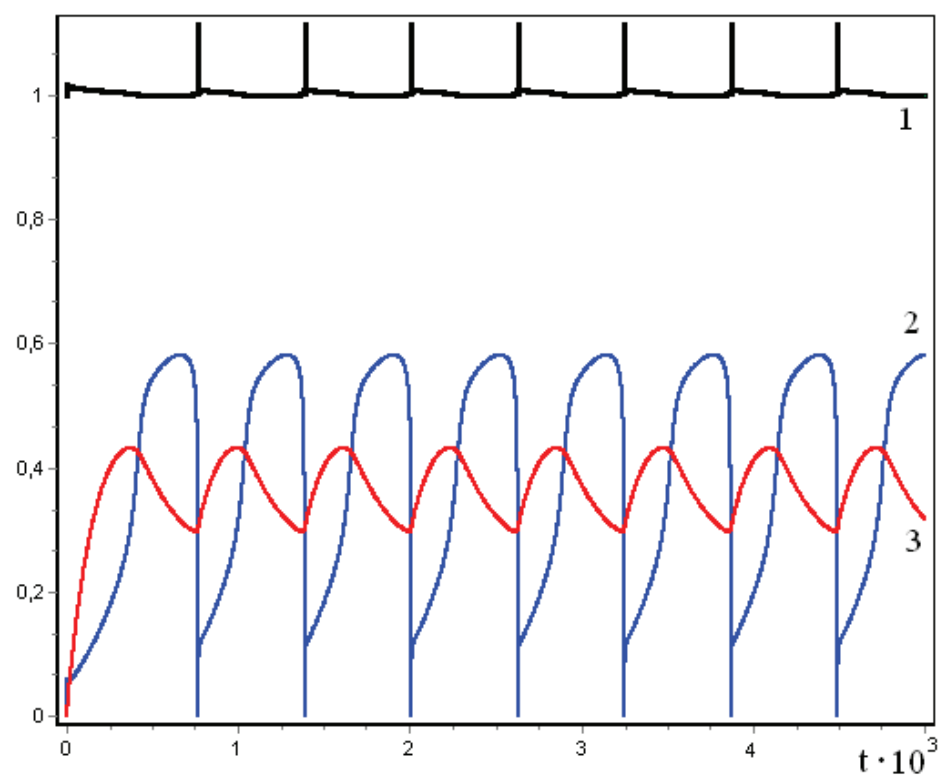

Figure 7. Oscillations in system (6.2) at $\gamma=65, \beta=0.6, s=4.5, D a=1, k_{1}=0.12$, $k_{-1}=0.01, k_{3}=0.0032, k_{-3}=0.002 ;(1) y(t),(2) x_{1}(t)$ and $(3) x_{2}(t)$. 


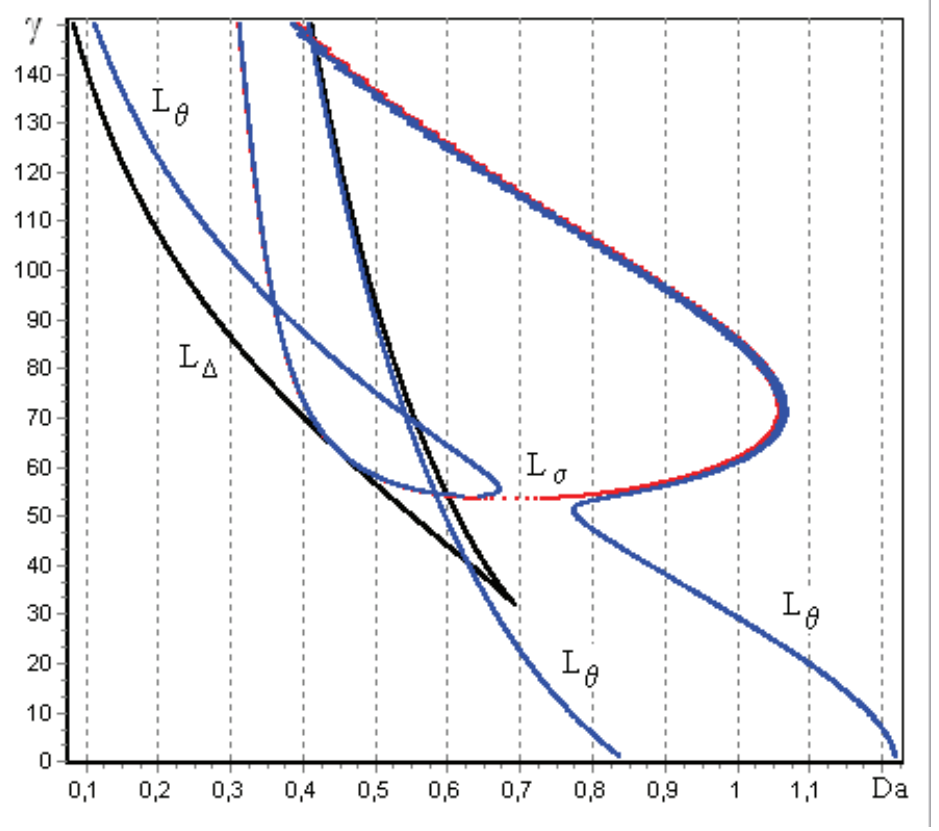

FiguRE 8. Parametric portrait of autocatalytic oscillator (6.2) on the $(D a, \gamma)$ plane: $\beta=0.6, s=1, k_{1}=0.12, k_{-1}=0.01, k_{3}=0.0032, k_{-3}=0.002$.

The use of modern mathematical methods and software allows us to advance substantially in an analysis of the whole diversity of the nonlinear properties of dynamic systems. As concerns the state of the problem, two directions can be indicated. The first one is works with fairly elaborate mathematical analysis methods (Lyapunov coefficients, nonlocal bifurcations, etc.) aimed at more deeply studying a particular model. A large diversity of phase portraits is then often obtained in very narrow parameter regions, which can be considered insignificant from the point of view of engineering. The second direction of works concerned with parametric analysis can be characterized as studies "in breadth" [22, 25]. In these works, the most significant rearrangements of phase portraits are identified, the principal bifurcation curves are constructed in various planes of physicochemical parameters, and the whole parametric analysis technology is given the form of an information-computational system, which is a necessary and effective tool for mathematical modeling of thermokinetic processes.

This work contains the results of an analysis of the influence of parameters on the dynamic characteristics of particular basic thermokinetic models rather than describes the technique of a mathematical study. Parametric analysis includes the determination of steady states, their stability, the dependence of stationary characteristics on parameters, and the construction of stationary state bifurcation lines, parametric and phase portraits, and time dependences. The results obtained are not only of methodological importance (they fairly completely describe the special features of several basic thermokinetic models) but also of interest for practical applications. The explicit equations for bifurcation curves in dimensionless parameter planes can be used to construct these curves also in dimensional thermophysical, geometric, and kinetic parameter planes corresponding to particular geometric, thermophysical, and kinetic characteristics of real exothermic processes.

Acknowledgements. The reported study was funded by RFBR according to the research project No. 15-01-00277. 


\section{References}

[1] R. Aris. Chemical reactors and some bifurcation phenomena. Annals of the N.Y. Acad. of Sci., 316 (1979), $314-331$.

[2] G. Eigenberger Kinetics instabilities in heterogeneously catalyzed reactions. Chem. Eng. Sci., 33 (1978), No. 9, 12631268.

[3] V. Hlavacek, J. Votruba. Hysteresis and periodic activity behavior in catalytic reactors Adv. in Catalysis., 27 (1978), No. 1, 59-96.

[4] R. Imbihl, G. Ertl. Oscillatory kinetics in heterogenenous catalysis. Chem. Rev., 95 (1995), No. 3, $697-794$.

[5] V. P. Zhdanov. Periodic perturbation of the kinetics of heterogeneous catalytic reactions. Surf. Sci. Rep. 55 (2004), No.1, 1-48.

[6] V. I. Bykov, S.B. Tsybenova. Nonlinear models of chemical kinetics. KRASAND, Moscow, 2011 [in Russian].

[7] V. V. Azatyan. Specific features of nonisothermal chain reactions and new theoretical aspects. Kinet. Catal., 40 (1999), No. 6, 812-834.

[8] V. V. Azatyan, Z. S. Andrianova, A. N. Ivanova. The role played by chain avalanches in the developed burning of hydrogen mixtures with oxygen and air at atmospheric pressure. Russ. J. Phys. Chem., 80 (2006), No. 7, $1044-1049$.

[9] V. I. Bykov. About simple models of oscillating catalytic reactions. Russ. J. Phys. Chem., 54 (1985), No. 11, $2712-2716$.

[10] M. M. Slinko, N. I. Jaeger. Oscillating heterogeneous catalytic systems. Elsevier, Amsterdam, 1994.

[11] V. I. Bykov. Modeling of chemical reactions in chemical kinetics. KomKniga, Moscow, 2006. [in Russian].

[12] G. S. Yablonski, V. I. Bykov, A. N. Gorban, V. I. Elokhin. Kinetic models of catalytic reactions. Elsevier, Amsterdam, 1991.

[13] G. S. Yablonskii, V. I. Bykov, A. N. Gorban'. Kinetic models of catalytic reactions. Nauka, Novosibirsk, 1983. [in Russian].

[14] G. S. Yablonskii, V. I. Bykov, V. I. Elokhin. Kinetics of model heterogeneous catalysis reactions. Nauka, Novosibirsk, 1984. [in Russian].

[15] V. I. Bykov, T. P. Pushkareva. Parametric analysis of kinetic models. 5. PT-diagram for the simplest autocatalytic oscillator. React. Kinet. Catal. Lett., 52 (1994), No. 1, 87-93.

[16] V. I. Bykov, T. P. Pushkareva. Parametric analysis of kinetic models. 7. Thermokinetic oscillator with autocatalysis. React. Kinet. Catal. Lett, 54 (1995), No. 1, 145-158.

[17] V. I. Bykov, S. B. Tsybenova. Semenov diagram as a steady-state stability criterion. Dokl. Phys. Chem. 374 (2000), No. 5, 196-199.

[18] V. I. Bykov, S. B. Tsybenova, M. G. Slin'ko. Andronov-Hopf bifurcations in the Aris-Amundson model. Dokl. Phys. Chem., 378 (2001), No. 1-3, 134-137.

[19] V. I. Bykov, S. B. Tsybenova, M. G. Slin'ko. Imperfectly stirred continuous reactor dynamics. Dokl. Chem. 378 (2001), No. 4-6, 298-301.

[20] G. A. Chumakov, M. G. Slin'ko, V. D. Belyaev. Complex changes in the rate of a heterogeneous catalytic reaction. Dokl. Akad. Nauk SSSR. 253 (1980), No. 3, 653-658.

[21] G. A. Chumakov, M. G. Slin'ko. Kinetic turbulence (chaos) of the hydrogen-oxygen interaction rate on metal-catalysts. Dokl. Akad. Nauk SSSR. 266 (1982), No. 5, 1194-1198.

[22] G. A. Chumakov, N. A. Chumakova. Relaxation oscillations in catalytic hydrogen oxidation including a chase on french ducks. XV Int. Conf. on Chem. Reactors CHEMREACTOR-15. Boreskov Inst. of Catalysis, Novosibirsk, 2001, 75-78.

[23] V. I. Bykov, S. B. Tsybenova. A model of thermokinetic oscillations on the surface of a catalyst. Russ. J. Phys. Chem., 77 (2003), No. 9, 1402-1405.

[24] V. I. Bykov, S. B. Tsybenova. Parametric analysis of the models of a stirred tank reactor and a tube reactor. Combustion, Explosion and Shock Waves, 37 (2001), No. 6, 634-640.

[25] V. I. Bykov, S. B. Tsybenova. Parametric analysis of the continuous stirred tank reactor model. Theor. Found. Chem. Eng., 37 (2003), No. 1, 59-69.

[26] M. Kholodniok, A. Klič, M. Kubiček, M. Marek. Methods of Analysis of Nonlinear Dynamical Models. Acad., Prague, 1986. [Mir, Moscow, 1991). 